\title{
ON THE DARBOUX INTEGRABILITY OF THE HINDMARSH-ROSE BURSTER
}

\author{
JAUME LLIBRE ${ }^{1}$ AND CLÀUDIA VALLS ${ }^{2}$
}

\begin{abstract}
We study the Hindmarsh-Rose burster which can be described by the differential system

$$
\dot{x}=y-x^{3}+b x^{2}+I-z, \quad \dot{y}=1-5 x^{2}-y, \quad \dot{z}=\mu\left(s\left(x-x_{0}\right)-z\right),
$$

where $b, I, \mu, s, x_{0}$ are parameters. We characterize all its invariant algebraic surfaces and all its exponential factors for all values of the parameters. We also characterize its Darboux integrability in function of the parameters.
\end{abstract}

\section{IntRoduction AND STATEMENT OF THE MAIN RESUlts}

One of the most studied three-dimensional ordinary differential systems that appear as a reduction of the conductance-based in the Hodgkin-Huxley model for neural spiking [11] is the Hindmarsh-Rose model [10] which can be written as:

$$
\begin{aligned}
& \dot{x}=y-x^{3}+b x^{2}+I-z, \\
& \dot{y}=1-5 x^{2}-y, \\
& \dot{z}=\mu\left(s\left(x-x_{0}\right)-z\right),
\end{aligned}
$$

where $b, I, \mu, s, x_{0}$ are parameters and the dot indicates derivative with respect to the time $t$.

The success of system (1) comes, first from its simplicity since it is just a differential system in $\mathbb{R}^{3}$ with a polynomial nonlinearity containing only five parameters, and second its ability to qualitatively capture the three main dynamical behaviors displayed by real neurons, namely quiescence, tonic spiking and bursting. Many papers have investigated the dynamics which takes place in system (1) when we vary one or more of its parameters, see for instance [2, 8, 9, 12, 13, 15, 22, 23, 24, 25, 26]. Among these amount of papers, none of them study its integrability.

The main aim of this paper is to study the existence of first integrals of system (1). The vector field $X$ associated to system (1) is

$$
X=\left(y-x^{3}+b x^{2}+I-z\right) \frac{\partial}{\partial x}+\left(1-5 x^{2}-y\right) \frac{\partial}{\partial y}+\mu\left(s\left(x-x_{0}\right)-z\right) \frac{\partial}{\partial z} .
$$

Let $U \subset \mathbb{R}^{3}$ be an open set. We say that the non-constant function $f: \mathbb{R}^{3} \rightarrow \mathbb{R}$ is a first integral of the vector field $X$ on $U$, if $F(x(t), y(t), z(t))=$ constant for all

2010 Mathematics Subject Classification. Primary 34C05, 34A34, 34C14.

Key words and phrases. polynomial integrability, rational integrability, Darboux polynomials, Darboux first integrals, invariant algebraic surfaces, exponential factors, Hindmarsh-Rose burster. 
values of $t$ for which the solution $(x(t), y(t), z(t))$ of $X$ is defined on $U$. Clearly $f$ is a first integral of $X$ on $U$ if and only if $X f=0$ on $U$.

The aim of this paper is to study the existence of first integrals of system (1) that can be described by functions of Darboux type (see (5)). Note that one of the main tools for studying the dynamics of the differential system (1) is to know the existence of first integrals for some values of the parameters $b, I, \mu, s, x_{0}$. In general, for a given differential system it is difficult to determine the existence or nonexistence of first integrals.

First we study the existence of first integrals of system (1) given by polynomials.

A polynomial first integral $f=f(x, y, z)$ of system (1) is a polynomial in the variables $x, y$ and $z$ such that

$$
\left(y-x^{3}+b x^{2}+I-z\right) \frac{\partial f}{\partial x}+\left(1-5 x^{2}-y\right) \frac{\partial f}{\partial y}+\mu\left(s\left(x-x_{0}\right)-z\right) \frac{\partial f}{\partial z}=0 .
$$

The first main result is the following.

Theorem 1. System (1) with $\mu \neq 0$ has no polynomial first integrals. If $\mu=0$ then the unique polynomial first integrals are polynomials in the variable $z$.

The proof of Theorem 1 is given in section 2 .

A rational first integral of system (1) is a rational function $f$ satisfying (2).

Theorem 2. System (1) with $\mu \neq 0$ has no rational first integrals. If $\mu=0$ then the unique rational first integrals are rational functions in the variable $z$.

The proof of Theorem 2 is given in section 4 .

For proving Theorem 2 we will use the Darboux theory of integrability. The Darboux theory of integrability in dimension 3 is based on the existence of invariant algebraic surfaces (or Darboux polynomials). For more details see [5, 6, 7]. This theory is one of the best theories for studying the existence of first integrals for the polynomial differential systems.

A Darboux polynomial of system (1) is a polynomial $f \in \mathbb{C}[x, y, z] \backslash \mathbb{C}$ such that

$$
\left(y-x^{3}+b x^{2}+I-z\right) \frac{\partial f}{\partial x}+\left(1-5 x^{2}-y\right) \frac{\partial f}{\partial y}+\mu\left(s\left(x-x_{0}\right)-z\right) \frac{\partial f}{\partial z}=K f,
$$

for some polynomial

$$
K=\alpha_{0}+\alpha_{1} x+\alpha_{2} y+\alpha_{3} z+\alpha_{4} x^{2}+\alpha_{5} x y+\alpha_{6} x z+\alpha_{7} y^{2}+\alpha_{8} y z+\alpha_{9} z^{2},
$$

called the cofactor of $f$. Note that $f=0$ is an invariant algebraic surface for the flow of system (1) and a polynomial first integral is a Darboux polynomial with zero cofactor.

Theorem 3. The unique irreducible Darboux polynomial with non-zero cofactor for system (1) exists for $s=0$ and $\mu \neq 0$, and in this case the Darboux polynomial is $z$ and the cofactor is $-\mu$. 
The proof of Theorem 3 is given in section 3 .

An exponential factor $F(x, y, z)$ of system (1) is a function of the form $F=$ $\exp \left(g_{0} / g_{1}\right) \notin \mathbb{C}$ with $g_{0}, g_{1} \in \mathbb{C}[x, y, z]$ coprime satisfying that

$$
\left(y-x^{3}+b x^{2}+I-z\right) \frac{\partial F}{\partial x}+\left(1-5 x^{2}-y\right) \frac{\partial F}{\partial y}+\mu\left(s\left(x-x_{0}\right)-z\right) \frac{\partial F}{\partial z}=L F,
$$

for some polynomial $L=L(x, y, z)$ of degree at most 2, called the cofactor of $F$.

Theorem 4. The following statements hold for system (1).

(i) If $\mu \neq 0$ system (1) has the exponential factors $e^{y}, e^{z}$, $e^{z^{2}}$ with cofactors $1-5 x^{2}-y, \mu\left(s\left(x-x_{0}\right)-z\right)$ and $2 \mu z\left(s\left(x-x_{0}\right)-z\right)$, respectively; and also exponential of linear combinations of $y, z$ and $z^{2}$.

(ii) If $\mu=0$ system (1) has the exponential factors $e^{y}, e^{P(z) / Q(z)}$ where $P, Q$ are polynomials with cofactors $1-5 x^{2}-y$ and 0 , respectively and also exponential of linear combinations of all the exponents in the previous exponential factors.

The proof of Theorem 4 is given in section 5 .

A Darboux first integral $G$ of system (1) is a first integral of the form

$$
G_{=} f_{1}^{\lambda_{1}} \cdots f_{p}^{\lambda_{p}} F_{1}^{\mu_{1}} \cdots F_{q}^{\mu_{q}}
$$

where $f_{1}, \ldots, f_{p}$ are Darboux polynomials and $F_{1}, \ldots, F_{q}$ are exponential factors and $\lambda_{j}, \mu_{k} \in \mathbb{C}$ for $j=1, \ldots, p$ and $k=1, \ldots, q$.

Theorem 5. System (1) with $\mu \neq 0$ has no Darboux first integrals. If $\mu=0$ then the unique Darboux first integrals are Darboux functions in the variable $z$.

The proof of Theorem 5 is given in section 6 .

\section{Polynomial first integrals: Proof of Theorem 1}

Let $f$ be a polynomial first integral of system (1). Without loss of generality we can assume that it has no constant term. Then $f$ satisfies (2). We write $f$ as $f=\sum_{j=0}^{n} f_{j}(x, y, z)$ where each $f_{j}$ is a homogeneous polynomial of degree $j$ in each variables $x, y$ and $z$. We can assume that $f_{n} \neq 0$ with $n>0$. We have that the terms of degree $n+2$ in (2) satisfy

$$
-x^{3} \frac{\partial f_{n}}{\partial x}=0 \text { that is } f_{n}=f_{n}(y, z) .
$$

Computing the terms of degree $n+1$ in (2) we get

$$
-x^{3} \frac{\partial f_{n-1}}{\partial x}-5 x^{2} \frac{\partial f_{n}}{\partial y}=0, \quad \text { that is }-x \frac{\partial f_{n-1}}{\partial x}=5 \frac{\partial f_{n}}{\partial y} .
$$

Since $f_{n}$ do not depend on $x$ we must have $\partial f_{n} / \partial y=0$ and $\partial f_{n-1} / \partial x=0$, that is $f_{n}=f_{n}(z)$ and $f_{n-1}=f_{n-1}(y, z)$.

Now computing the terms of degree $n$ in (2) we obtain

$$
-x^{3} \frac{\partial f_{n-2}}{\partial x}-5 x^{2} \frac{\partial f_{n-1}}{\partial y}+\mu(s x-z) \frac{d f_{n}}{d z}=0 .
$$


Note that if $\mu \neq 0$ then $\mu(s x-z)$ must be divisible by $x^{2}$ which is not possible and thus $d f_{n} / d z=0$ which yields $f_{n}$ is a constant, in contradiction with the fact that $f$ is a first integral. Hence $\mu=0$. In this case proceeding as above, after simplifying by $x^{2}$ and taking into account that $f_{n-1}$ does not depend on $x$ we get that $\partial f_{n-1} / \partial y=0$ and $\partial f_{n-2} / \partial x=0$, that is $f_{n-1}=f_{n-1}(z)$ and $f_{n-2}=f_{n-2}(y, z)$. Proceeding inductively we conclude that $f=\sum_{j=0}^{n} f_{j}(z)$, that is, $f$ is a polynomial in the variable $z$. This concludes the proof of Theorem 1 .

\section{Darboux polynomials with non-Zero cofactor: Proof of Theorem 3}

We consider a Darboux polynomial $f$ with non-zero cofactor $K$ as in (4). We denote by $\mathbb{N}$ the set of all positive integers.

We first prove three preliminary results.

Lemma 6. $\alpha_{5}=\alpha_{6}=\alpha_{7}=\alpha_{8}=\alpha_{9}=0$ and $\alpha_{4}=m$ with $m \in \mathbb{N} \cup\{0\}$.

Proof. We write $f=\sum_{j=0}^{n} f_{j}(x, y, z)$ where each $f_{j}$ is a homogeneous polynomial of degree $j$ in each variables $x, y$ and $z$. Without loss of generality we can assume that $f_{n} \neq 0$ and $n>0$.

We have that the terms of degree $n+2$ in (3) satisfy

$$
x^{3} \frac{\partial f_{n}}{\partial x}=\left(\alpha_{4} x^{2}+\alpha_{5} x y+\alpha_{6} x z+\alpha_{7} y^{2}+\alpha_{8} y z+\alpha_{9} z^{2}\right) f_{n} .
$$

Solving the differential equation in (7) we have

$$
f_{n}=K_{n}(y, z) x^{\alpha_{4}} \exp \left(-\frac{\alpha_{5} y+\alpha_{6} z}{x}-\frac{\alpha_{7} y^{2}+\alpha_{8} y z+\alpha_{9} z^{2}}{2 x^{2}}\right),
$$

where $K_{n}$ is any function in the variables $y$ and $z$. Since $f_{n}$ must be a homogeneous polynomial of degree $n$ we must have $\alpha_{4}=m, m \in \mathbb{N} \cup\{0\}$ and $\alpha_{5}=\alpha_{6}=\alpha_{7}=$ $\alpha_{8}=\alpha_{9}=0$. This completes the proof of the lemma.

Lemma 7. $\alpha_{2}=0$.

Proof. We write $f=\sum_{j=0}^{n} f_{j}(x, z) y^{j}$ where each $f_{j}$ is a polynomial in the variables $x, z$. The terms of $y^{n+1}$ in (3) satisfy

$$
\frac{\partial f_{n}}{\partial x}=\alpha_{2} f_{n}
$$

Solving this differential equation we obtain

$$
f_{n}=K_{n}(z) \exp \left(\alpha_{2} x\right),
$$

where $K_{n}$ is any function in the variable $z$. Since $f_{n}$ must be a polynomial we must have $\alpha_{2}=0$ or $K_{n}=0$. If $K_{n}=0$, then $f_{n}=0$, and consequently $f=f(x, z)$. Then, from $(3)$

$$
y \frac{\partial f}{\partial x}=\alpha_{2} y f \text { that is } f=K(z) e^{\alpha_{2} x},
$$

for some function $K$ of $z$. Since $f$ must be a polynomial we get $\alpha_{2}=0$. This completes the proof of the lemma. 
Lemma 8. $\alpha_{3}=0$.

Proof. We write $f=\sum_{j=0}^{n} f_{j}(x, y) z^{j}$ where each $f_{j}$ is a polynomial in the variables $x$ and $y$. The terms of $z^{n+1}$ in (3) satisfy

$$
\frac{\partial f_{n}}{\partial x}=-\alpha_{3} f_{n}
$$

Solving this differential equation we obtain

$$
f_{n}=K_{n}(y) \exp \left(-\alpha_{3} z\right)
$$

where $K_{n}$ is any function in the variable $y$. Since $f_{n}$ must be a polynomial we must have $\alpha_{3}=0$ or $K_{n}=0$. If $K_{n}=0$, then $f_{n}=0$, and consequently $f=f(x, y)$. Then, from $(3)$

$$
-z \frac{\partial f}{\partial x}=\alpha_{3} z f \quad \text { that is } f=K(y) e^{-\alpha_{3} x},
$$

for some function $K$ of $y$. Since $f$ must be a polynomial we get $\alpha_{3}=0$. This completes the proof of the lemma.

In view of Lemmas 6,7 and 8 we have that

$$
K=\alpha_{0}+\alpha_{1} x+m x^{2}, \quad m \in \mathbb{N} \cup\{0\} .
$$

Now for simplicity in the computations we introduce the following weight change of variables

$$
x=\lambda^{-1} X, \quad y=Y, \quad z=Z, \quad t=\lambda^{2} T
$$

with $\lambda \in \mathbb{R} \backslash\{0\}$ and system (1) becomes

$$
\begin{aligned}
& X^{\prime}=-X^{3}+b \lambda X^{2}+\lambda^{3}(Y+I-Z), \\
& Y^{\prime}=-5 X^{2}+\lambda^{2}(1-Y), \\
& Z^{\prime}=\lambda \mu s X-\lambda^{2} \mu\left(s x_{0}+Z\right),
\end{aligned}
$$

where the prime denotes derivative of the variables with respect to $T$.

Let $g(x, y, z)$ be a Darboux polynomial of system (1) with cofactor $K$ given in (8). We use the transformation (9) and setting $G(X, Y, Z)=\lambda^{n} g\left(\lambda^{-1} X, Y, Z\right)$ where $n$ is the degree of $g$ and $k=\lambda^{2} K\left(\lambda^{-1} X\right)=m X^{2}+\lambda \alpha_{1} X+\lambda^{2} \alpha_{0}$. Then we have

$$
\frac{d G}{d T}=\lambda^{n+2} \frac{d g}{d t}=\lambda^{n+2} K g=k G .
$$

Assume $G=\sum_{i=0}^{n} \lambda^{i} G_{i}$, where $G_{i}$ is a homogeneous polynomial in the variables $X, Y, Z$ with degree $n-i$ for $i=0, \ldots, n$. Clearly $g=G_{\mid \lambda=1}$. By definition of a Darboux polynomial we have

$$
\begin{aligned}
& \left(-x^{3}+b \lambda x^{2}+\lambda^{3}(y+I-z)\right) \sum_{i=0}^{n} \lambda^{i} \frac{\partial G_{i}}{\partial x}+\left(-5 x^{2}+\lambda^{2}(1-y)\right) \sum_{i=0}^{n} \lambda^{i} \frac{\partial G_{i}}{\partial y} \\
& +\left(\lambda \mu s x-\lambda^{2} \mu\left(s x_{0}+z\right)\right) \sum_{i=0}^{n} \lambda^{i} \frac{\partial G_{i}}{\partial z}=\left(m x^{2}+\lambda \alpha_{1} x+\lambda^{2} \alpha_{0}\right) \sum_{i=0}^{n} \lambda^{i} G_{i}
\end{aligned}
$$


where now we use $x, y, z$ instead of $X, Y, Z$. Equating the terms with $\lambda^{i}$ for $i=$ $0, \ldots, n+2$ we get

$$
L\left[G_{0}\right]-m x^{2} G_{0}=0 \quad \text { where } \quad L=-x^{3} \frac{\partial}{\partial x}-5 x^{2} \frac{\partial}{\partial y}
$$

and

$$
\begin{aligned}
L\left[G_{1}\right]-m x^{2} G_{1}= & -b x^{2} \frac{\partial G_{0}}{\partial x}-\mu s x \frac{\partial G_{0}}{\partial z}+\alpha_{1} x G_{0} \\
L\left[G_{2}\right]-m x^{2} G_{2}= & -b x^{2} \frac{\partial G_{1}}{\partial x}-\mu s x \frac{\partial G_{1}}{\partial z}-(1-y) \frac{\partial G_{0}}{\partial y}+\mu\left(s x_{0}+z\right) \frac{\partial G_{0}}{\partial z} \\
& +\alpha_{1} x G_{1}+\alpha_{0} G_{0}, \\
L\left[G_{j}\right]-m x^{2} G_{j}= & -b x^{2} \frac{\partial G_{j-1}}{\partial x}-\mu s x \frac{\partial G_{j-1}}{\partial z}-(1-y) \frac{\partial G_{j-2}}{\partial y}+\mu\left(s x_{0}+z\right) \frac{\partial G_{j-2}}{\partial z} \\
& -(y+I-z) \frac{\partial G_{j-3}}{\partial x}+\alpha_{1} x G_{j-1}+\alpha_{0} G_{j-2},
\end{aligned}
$$

for $j=3, \ldots, n+2$.

Solving (11) we get

$$
G_{0}=x^{-m} \tilde{G}_{0}(z, 5 \log x-y) .
$$

In order that $G_{0}$ be a homogeneous polynomial of degree $n$ we get $m=0$ and

$$
G_{0}=a_{0} z^{n} \quad \text { with } \quad a_{0} \in \mathbb{C} \backslash\{0\} .
$$

Substituting $G_{0}$ into the first equation (12) and simplifying by $x$ we get

$$
-x\left(x \frac{\partial G_{1}}{\partial x}-5 \frac{\partial G_{1}}{\partial y}\right)=\alpha_{1} a_{0} z^{n}-\mu s n a_{0} z^{n-1} .
$$

Evaluating (13) on $x=0$ and taking into account that $a_{0} n \neq 0$ we get $\alpha_{1}=0$ and $\mu s=0$. Then again from (13) we get that

$$
x \frac{\partial G_{1}}{\partial x}-5 \frac{\partial G_{1}}{\partial y}=0 \text { that is } G_{1}=G_{1}(z, 5 \log x-y) .
$$

Since $G_{1}$ must be a polynomial of degree $n-1$ we obtain $G_{1}=a_{1} z^{n-1}$ where $a_{1} \in \mathbb{C}$.

Substituting $G_{0}$ and $G_{1}$ into the second equation (12) taking into account that $\alpha_{1}=\mu s=0$ and we get

$$
-x^{2}\left(x \frac{\partial G_{2}}{\partial x}-5 \frac{\partial G_{2}}{\partial y}\right)=a_{0}\left(\mu n+\alpha_{0}\right) z^{n} .
$$

We consider two different cases.

Case 1: $\mu=0$. In this case, evaluating (15) on $x=0$ we get $\alpha_{0}=0$, but then $K=0$ in contradiction with the fact that $f$ is a Darboux polynomial with non-zero cofactor. Case 2: $\mu \neq 0$. Since $\mu s=0$ then $s=0$. Evaluating (15) on $x=0$ we obtain $\alpha_{0}=-\mu n$, and proceeding as we $\operatorname{did}$ for $G_{1}$ we get $G_{2}=a_{2} z^{n-2}$ with $a_{2} \in \mathbb{C}$. 
Substituting $G_{0}, G_{1}$ and $G_{2}$ into equation (12) with $j=3$ and taking into account that $\alpha_{1}=\mu s=0, \alpha_{0}=-\mu n$ we get

$$
-x^{2}\left(x \frac{\partial G_{3}}{\partial x}-5 \frac{\partial G_{3}}{\partial y}\right)=-\mu a_{1} z^{n-1} .
$$

Evaluating (16) on $x=0$ and using that $\mu \neq 0$ we get $a_{1}=0$, that is $G_{1}=0$. Furthermore, $G_{3}=a_{3} z^{n-3}$ with $a_{3} \in \mathbb{C}$.

Proceeding inductively we get $G_{i}=0$ for $i=1, \ldots, n+2, G_{0}=a_{0} z^{n}$ and $k=$ $\alpha_{0}=-\mu n$. Then $g=a_{0} z^{n}$ and $K=-\mu n$. In short, the unique irreducible Darboux polynomial of system (1) is $z$ and its cofactor is $-\mu$. This concludes the proof of Theorem 3.

\section{Proof of Theorem 2}

To prove Theorem 2 we recall two auxiliary results. The first was proved in [7] while the second was proved in [16].

Lemma 9. Let $f$ be a polynomial and $f=\prod_{j=1}^{s} f_{j}^{\alpha_{j}}$ its decomposition into irreducible factors in $\mathbb{C}[x, y, z]$. Then $f$ is a Darboux polynomial if and only if all the $f_{j}$ are Darboux polynomials. Moreover, if $K$ and $K_{j}$ are the cofactors of $f$ and $f_{j}$, then $K=\sum_{j=1}^{s} \alpha_{j} K_{j}$

Lemma 10. The existence of a rational first integral for a polynomial differential system (1) implies the existence of a polynomial first integral, or the existence of two Darboux polynomials with the same non-zero cofactor.

The proof of Theorem 2 follows readily from Theorems 1 and 3 together with Lemmas 9 and 10.

\section{Exponential Factors: Proof of Theorem 4}

To prove Theorem 4 we will use the following known result whose proof and geometrical meaning is given in [3] for the plane systems and [17] and [18] for higher dimensional systems.

Proposition 11. The following statements hold.

(a) If $E=\exp \left(g_{0} / g_{1}\right)$ is an exponential factor for the polynomial system (1) and $g_{1}$ is not a constant polynomial, then $g_{1}=0$ is an invariant algebraic curve.

(b) Eventually $e^{g_{0}}$ can be exponential factors, coming from the multiplicity of the infinite invariant straight line.

The following result given in [3] and [17] characterizes the algebraic multiplicity of an invariant algebraic surface using the number of exponential factors of system (1) associated with this invariant algebraic surface. 
Theorem 12. Given an irreducible invariant algebraic surface $g_{1}=0$ of degree $m$ of system (1), it has algebraic multiplicity $k$ if and only if the vector field associated to system (1) has $k-1$ exponential factors of the form $\exp \left(g_{0, i} / g_{1}^{i}\right)$, where $g_{0, i}$ is a polynomial of degree at most $i m$, and $g_{0, i}$ and $g_{1}$ are coprime for $i=1, \ldots, k-1$.

In view of Theorem 12 if we prove that $e^{g_{0} / g_{1}}$ is not an exponential factor with degree $g_{0} \leq$ degree $g_{1}$, there are no exponential factors associated to the invariant algebraic surface $g_{1}=0$.

Proposition 13. System (1) with $\mu \neq 0$ has the exponential factors $e^{y}, e^{z}$, $e^{z^{2}}$ with cofactors $1-5 x^{2}-y, \mu\left(s\left(x-x_{0}\right)-z\right)$ and $2 \mu z\left(s\left(x-x_{0}\right)-z\right)$, respectively and also exponential of linear combinations of $y, z$ and $z^{2}$.

Proof. System (1) has the irreducible Darboux polynomial $z$ when $s=0$. Then in view of Proposition 11 system (1) can have an exponential factor of the form: either $E=\exp (g)$ with $g \in \mathbb{C}[x, y, z]$, or only when $s=0, E=\exp \left(h / z^{m}\right)$ with $m \geq 1$ and such that $h \in \mathbb{C}[x, y, z]$ is coprime with $z$ and the degree of $h$ is at most $m$.

We first prove that system (1) with $s=0$ has no exponential factors of the form $E=\exp \left(h / z^{m}\right)$.

Assume that system (1) with $s=0$ has an exponential factor of the form $E=$ $\exp \left(h / z^{m}\right)$ with $m \geq 1$ such that $z$ is coprime with $h \in \mathbb{C}[x, y, z]$. In view of Theorem 12 we can assume that $m=1$ and that $h$ has degree at most one (note that here $g_{1}=z$ has degree one). We write $h$ as a polynomial of degree one in the variables $x, y, z$ as follows

$$
h=a_{0}+a_{1} x+a_{2} y+a_{3} z .
$$

Clearly $h$ satisfies

$$
\left(y-x^{3}+b x^{2}+I-z\right) \frac{\partial h}{\partial x}+\left(1-5 x^{2}-y\right) \frac{\partial h}{\partial y}-\mu z \frac{\partial h}{\partial z}+\mu h=L z,
$$

where $L$ is a polynomial of degree two in the variables $x, y, z$. Let

$$
L=b_{0}+b_{1} x+b_{2} y+b_{3} z+b_{4} x^{2}+b_{5} x y+b_{6} x z+b_{7} y^{2}+b_{8} y z+b_{9} z^{2} .
$$

From (18) and using an algebraic manipulator it is easy to check that

$$
h=a_{3} z \quad \text { and } \quad L=0 .
$$

However this is not possible since $h$ is coprime with $z$. Hence this case is not possible.

In summary, if $\mu \neq 0$ and (1) has an exponential factor it must be of the form $E=\exp (g)$ with $g \in \mathbb{C}[x, y, z] \backslash \mathbb{C}$. In this case, $g$ satisfies

$$
\left(y-x^{3}+b x^{2}+I-z\right) \frac{\partial g}{\partial x}+\left(1-5 x^{2}-y\right) \frac{\partial g}{\partial y}+\mu\left(\left(s\left(x-x_{0}\right)-z\right)\right) \frac{\partial g}{\partial z}=L,
$$

where $L=L(x, y, z)$ is some polynomial of degree two in the variables $x, y$ and $z$ and that we can take as in (19). 
We write $g$ as $g=\sum_{j=0}^{n} g_{j}(x, y, z)$ where each $g_{j}$ is a homogeneous polynomial of degree $j$ in each variables $x, y$ and $z$ and $n>0$. We have that the terms of degree $n+2$ with $n \geq 3$ in (20) satisfy

$$
-x^{3} \frac{\partial g_{n}}{\partial x}=0, \text { that is } g_{n}=g_{n}(y, z) .
$$

Computing the terms of degree $n+1$ with $n \geq 3$ in (20) we get

$$
-x^{3} \frac{\partial g_{n-1}}{\partial x}-5 x^{2} \frac{\partial g_{n}}{\partial y}=0, \quad \text { that is }-x \frac{\partial g_{n-1}}{\partial x}=5 \frac{\partial g_{n}}{\partial y} .
$$

Since $g_{n}$ do not depend on $x$ we must have $\partial g_{n} / \partial y=0$ and $\partial g_{n-1} / \partial x=0$ that is $g_{n}=g_{n}(z)$ and $g_{n-1}=g_{n-1}(y, z)$.

Now computing the terms of degree $n$ with $n \geq 3$ in (20) we obtain

$$
-x^{3} \frac{\partial g_{n-2}}{\partial x}-5 x^{2} \frac{\partial g_{n-1}}{\partial y}+\mu(s x-z) \frac{d g_{n}}{d z}=0
$$

Note that since $\mu \neq 0$ then $\mu(s x-z)$ must be divisible by $x^{2}$ which is not possible and thus $d g_{n} / d z=0$ which yields $g_{n}$ is a constant, in contradiction with the fact that $n \geq 3$. Then, we must have $n \leq 2$. In this case $g$ is a polynomial of degree two in its variables that we write it as

$$
g=a_{0}+a_{1} x+a_{2} y+a_{3} z+a_{4} x^{2}+a_{5} x y+a_{6} x z+a_{7} y^{2}+a_{8} y z+a_{9} z^{2} .
$$

Imposing that $g$ satisfies (20) with $L$ as in (19) using an algebraic manipulator such as mathematica we get

$$
g=a_{0}+a_{2} y+a_{3} z+a_{9} z^{2}
$$

and

$$
L=a_{2}\left(1-5 x^{2}-y\right)+a_{3} \mu\left(s\left(x-x_{0}\right)-z\right)+2 \mu a_{9} z\left(s\left(x-x_{0}\right)-z\right) .
$$

This concludes the proof of the proposition.

Now we have to study the case $\mu=0$. This is the content of the following result.

Proposition 14. System (1) with $\mu=0$ has the exponential factors $e^{y}, e^{P(z) / Q(z)}$ where $P, Q$ are polynomials with cofactors $1-5 x^{2}-y$ and 0 , respectively and also exponential of linear combinations of all the exponents in the previous exponential factors.

Proof. System (1) has the polynomial first integral $z$ when $\mu=0$. Then in view of Proposition 11 system (1) can have an exponential factor of the form: either $E=\exp (g)$ with $g \in \mathbb{C}[x, y, z]$, or $E=\exp \left(h / z^{m}\right)$ with $m \geq 1$ and such that $h \in \mathbb{C}[x, y, z]$ is coprime with $z$ and has degree at mos $m$

We first prove that system (1) with $\mu=0$ has no exponential factors of the form $E=\exp \left(h / z^{m}\right)$.

Assume that system (1) with $\mu=0$ has an exponential factor of the form $E=$ $\exp \left(h / z^{m}\right)$ with $m \geq 1$ such that $z$ is coprime with $h \in \mathbb{C}[x, y, z]$. In view of Theorem 
12 we can assume that $m=1$ and that $h$ has degree at most one. We write $h$ as a polynomial of degree one in the variables $x, y, z$ as in (17). Clearly, $h$ satisfies

$$
\left(y-x^{3}+b x^{2}+I-z\right) \frac{\partial h}{\partial x}+\left(1-5 x^{2}-y\right) \frac{\partial h}{\partial y}=L z
$$

where $L$ is a polynomial of degree two in the variables $x, y, z$. Setting $L$ as in (19) in (24) and working with an algebraic manipulator we conclude that

$$
h=a_{0}+a_{3} z \quad \text { and } \quad L=0 .
$$

Now assume that system (1) with $\mu=0$ has an exponential factor of the form $E=\exp (g)$ with $g \in \mathbb{C}[x, y, z] \backslash \mathbb{C}$. In this case, $g$ satisfies

$$
\left(y-x^{3}+b x^{2}+I-z\right) \frac{\partial g}{\partial x}+\left(1-5 x^{2}-y\right) \frac{\partial g}{\partial y}=L,
$$

where $L=L(x, y, z)$ is some polynomial of degree two in the variables $x, y, z$ and that we can take as in (19).

We write $g$ as $g=\sum_{j=0}^{n} g_{j}(x, y, z)$ where each $g_{j}$ is a homogeneous polynomial of degree $j$ in each variables $x, y$ and $z$. We have that the terms of degree $n+2$ with $n \geq 3$ in (25) satisfy

$$
-x^{3} \frac{\partial g_{n}}{\partial x}=0 \text { that is } g_{n}=g_{n}(y, z) .
$$

Computing the terms of degree $n+1$ with $n \geq 3$ in $(25)$ we get

$$
-x^{3} \frac{\partial g_{n-1}}{\partial x}-5 x^{2} \frac{\partial g_{n}}{\partial y}=0, \quad \text { that is } \quad-x \frac{\partial g_{n-1}}{\partial x}=5 \frac{\partial g_{n}}{\partial y} .
$$

Since $g_{n}$ do not depend on $x$ we must have $\partial g_{n} / \partial y=0$ and $\partial g_{n-1} / \partial x=0$ that is $g_{n}=g_{n}(z)$ and $g_{n-1}=g_{n-1}(y, z)$.

Now computing the terms of degree $n$ with $n \geq 3$ in (25) we obtain

$$
-x^{3} \frac{\partial g_{n-2}}{\partial x}-5 x^{2} \frac{\partial g_{n-1}}{\partial y}=0 .
$$

Now proceeding as above, after simplifying by $x^{2}$ and taking into account that $g_{n-1}$ does not depend on $x$, we get that $\partial g_{n-1} / \partial y=0$ and $\partial g_{n-2} / \partial x=0$ that is $g_{n-1}=$ $g_{n-1}(z)$ and $g_{n-2}=g_{n-2}(y, z)$. Proceeding inductively, we get

$$
g=G(z)+a_{2} z^{2}+a_{3} y+a_{4} z+a_{0}=\tilde{G}(z)+a_{3} y,
$$

where $G$ and $\tilde{G}$ are polynomials in the variable $z$ and $a_{3} \in \mathbb{C}$. Moreover, it follows from (25) that $L=a_{3}\left(1-5 x^{2}-y\right)$. This completes the proof of the proposition for $n \geq 3$.

If $n<3$ then $g$ can be written as in (21). Solving (25) with $g$ and $L$ as in (19) we get $g$ as in $(22)$ and $L=a_{2}\left(1-5 x^{2}-y\right)$. So the proposition is proved. 


\section{Proof of Theorem 5}

In order to proof Theorem 5 we need the following result whose proof is given in [7] and [17].

Theorem 15. Suppose that system (1) admits p Darboux polynomials and with cofactors $K_{i}$ and $q$ exponential factors $F_{j}$ with cofactors $L_{j}$. Then there exists $\lambda_{j}, \mu_{j} \in \mathbb{C}$ not all zero such that

$$
\sum_{i=1}^{q} \lambda_{k} K_{i}+\sum_{i=1}^{q} \mu_{i} L_{i}=0
$$

if and only if the function $G$ given in (5) (called of Darboux type) is a first integral of system (1).

In view of Theorem 15 to characterize the Darboux first integrals we need to compute the Darboux polynomials and the exponential factors. We consider three cases.

Case 1: $\mu s \neq 0$ In this case, using Theorems 3 and 4 if $G$ is a Darboux first integral of system (1) it must be of the form (5) and the cofactors must satisfy

$$
\mu_{1}\left(1-5 x^{2}-y\right)+\mu_{2} \mu\left(s\left(x-x_{0}\right)-z\right)+2 \mu \mu_{3} z\left(s\left(x-x_{0}\right)-z\right)=0 .
$$

Solving this system we have $\mu_{1}=\mu_{2}=\mu_{3}=0$, which yields that $G$ is a constant, that is, there are no Darboux first integrals.

Case 2: $\mu \neq 0$ and $s=0$ In this case, using Theorems 3 and 4 if $G$ is a Darboux first integral of system (1) it must be of the form (5) and the cofactors must satisfy

$$
\mu_{1}\left(1-5 x^{2}-y\right)-\mu_{2} \mu z-2 \mu \mu_{3} z^{2}-\lambda_{1} \mu=0 .
$$

Solving this system we have $\mu_{1}=\mu_{2}=\mu_{3}=\lambda_{1}=0$, which yields that $G$ is a constant, that is, there are no Darboux first integrals.

Case 3: $\mu=0$ In this case, using Theorems 3 and 4 if $G$ is a Darboux first integral of system (1) it must be of the form (5) and the cofactors must satisfy

$$
\mu_{1}\left(1-5 x^{2}-y\right)+\mu_{2} 0=0 .
$$

Solving this system we have either $\mu_{1}=0$ which yields that $G$ is a function in the variable $z$. This concludes the proof of the theorem.

\section{ACKNOWLEDGEMENTS}

The first author is partially supported by a MINECO/FEDER grant MTM2008 03437, a CIRIT grant number 2009SGR-410, an ICREA Academia, and two grants FP7-PEOPLE-2012-IRSES 316338 and 318999. The second author was supported by Portuguese National Funds through FCT - Fundação para a Ciência e a Tecnologia within the project PTDC/MAT/117106/2010 and by CAMGSD. 


\section{REFERENCES}

[1] R. Abraham and J.E. Marsden, Foundations of Mechanics, Benjamin, Reading, Massachussets, 1978.

[2] V.N. Belykh, I.V. Belykh, M. Colding-Jorgensen and E. Mosekilde, Homoclinic bifurcations leading to the emergence of bursting oscillations in cell models, The European Physical Journal E: Soft Matter and Biological Physics 3 (2000), 205-219.

[3] C. Christopher, J. Llibre and J.V. Pereira, Multiplicity of invariant algebraic curves in polynomial vector fields, Pacific J. Math. 229 (2007), 63-117.

[4] G. Contopoulos and J. Seimenis, Application of the Prendergast method to a logarithmic potential, Astron. Astrophys. 227 (1990), 49-53.

[5] G. Darboux, Mémoire sur les équations différentielles du premier ordre et du premier degreé (Mélanges), Bull. Sci. Math. 2éme série 2 (1878), 60-96, 123-144, 151-200.

[6] G. Darboux, De l'emploi des solutions particulières algébriques dans l'intégration des systèmes d'équations différentielles algébriques, C. R. Math. Acad. Sci. Paris 86 (1878), 1012-1014.

[7] F. Dumortier, J. Llibre And J.C. ArtÉS, Qualitative theory of planar differential systems, Universitext, Springer-Verlag, Berlin, 2006.

[8] J.M. GonzÁLEZ-MirAnda, Observation of a continuous interior crisis in the Hindmarsh-Rose neuron model, Chaos 13 (2003), 845-852.

[9] J.M. GonzÁlez-Miranda, Complex bifurcation structures in the Hindmarsh-Rose neuron model, Int. J. Bifurcation and Chaos 17 (2007), 3071-3083.

[10] J.L. Hindmarsh And R.M. Rose, A model of neuronal bursting using three coupled first order differential equations, Proceedings of the Royal Society of London. Series B, Biological Sciences 221 (1984), 87-102. 246 (2009), 541-551.

[11] A.L. Hodgkin And A.F. HuXley, A quantitative description of membrane current and its application to conduction and excitation in nerve, J. Physiol.-London 117 (1952), 500-544.

[12] G. Innocenti and R. Genesio, On the dynamics of chaotic spiking-bursting transition in the Hindmarsh-Rose neuron, Chaos 19 (2009), 023124-8 pp.

[13] G. Innocenti, A. Morelli, R. Genesio And A. Torcini, Dynamical phases of the Hindmarsh-Rose neuronal model: Studies of the transition from bursting to spiking chaos, Chaos 17 (2007), 043128-11 pp.

[14] L. Jimenez-Lara And J. Llibre, The cored and logarithmic galactic potentials: Periodic orbits and integrability, J. Math. Phys. 53, (2012), 042901-12 pp.

[15] D. Linaro, A. Champneys, M. Desroches and M. Storace, Codimension-two homoclinic bifurcations underlying spike adding in the Hindmarsh-Rose burster, SIAM J. Appl. Dyn. Syst. 11 (2012), 939-962.

[16] J. Llibre And C. Valls, On the integrability of the Bianchi IX system, J. Math. Phys. 46 (2005), 072901-13 pp.

[17] J. Llibre And X. Zhang, Darboux theory of integrability in $\mathbb{C}^{n}$ taking into account the multiplicity, J. Differential Equations 246 (2009), 541-551.

[18] J. Llibre AND X. Zhang, Darboux theory of integrability for polynomial vector fields in $\mathbb{R}^{n}$ taking into account the multiplicity at infinity, Bull. Sci. Math. 133 (2009), 765-778.

[19] Y. PAPAPHILIPPOU AND J. LASKAR, Frequency map analysis and global dynamics in a two degrees of freedom galactic potential, Astron. Astrophys. 307 (1996), 426-449.

[20] G. Pucacco, D. Boccaletti and C. Belmonte, Quantitative predictions with detuned normal forms, Celes. Mech. Dyn. Astron. 102 (2008), 163-176.

[21] G. Pucacco, D. Boccaletti and C. Belmonte, Periodic orbits in the logarithmic potential, Astron. Astrophys. 489 (2008), 1055-1063.

[22] A. Silnikov And M. Kolomiets, Methods of the qualitative theory for the Hindmarsh-Rose model: a case study. a tutorial, Int. J. Bifurcation and Chaos 18 (2008), 2141-2168.

[23] M. Storace, D. Linaro and E. De Lange, The Hindmarsh-Rose neuron model: bifurcation analysis and piecewise-linear approximations, Chaos 18 (2008), 033128-10 pp. 
[24] D. TERMAN, Chaotic spikes arising from a model of bursting in excitable membranes, SIAM J. Appl. Math. 51 (1991), 1418-1450.

[25] D. TERMAn, The transition from bursting to continuous spiking in excitable membrane models, J. Nonlinear Science 2 (1992), 135-182.

[26] X.J. WANG, Genesis of bursting oscillations in the Hindmarsh-Rose model and homoclinicity to a chaotic saddle, Physica D 62 (1993), 263-274.

1 Departament de Matemàtiques, Universitat Autònoma de Barcelona, 08193 Bellaterra, Barcelona, Catalonia, Spain

E-mail address: jllibre@mat.uab.cat

2 Departamento de Matemática, Instituto Superior Técnico, Universidade Técnica De Lisboa, Av. Rovisco Pais 1049-001, LisboA, Portugal

E-mail address: cvalls@math.ist.utl.pt 